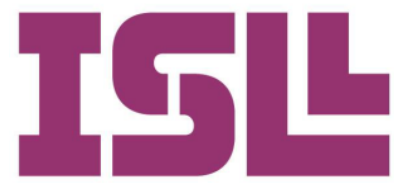

Número 8.

Julio de 2017

\section{Aplicación de un programa para mejorar la velocidad y comprensión de lectura en estudiantes universitarios}

\section{Implementation of a program to improve reading speed and comprehension of college students}

\author{
Miguel Barboza-Palomino \\ José Luis Ventura-León \\ Universidad Privada del Norte (Perú)
}

Pág. 49 a la 59

\section{Keywords}

reading programs, speed reading, college students, Latin Americans

\begin{abstract}
:
The effect of a program to improve reading speed and comprehension was assessed in students from a private peruvian university, for which a quasi-experimental design of longitudinal pretest-postest type was developed and using a protocol based on a methodology of reading program fast, study and memory, was selected for a total sample of 1181 college freshmen from Universidad Privada del Norte (UPN) who were studying on the 2014-II academic semester. Results show a statistically significant increase on reading speed, but not in reading comprehension; furthermore, the practical effect of the program is moderate. It is concluded that the program had a positive moderate effect on reading speed; however, it was not an effective proposal to improve reading comprehension.
\end{abstract}




\section{Introducción}

En el contexto latinoamericano, las universidades que buscan ser competitivas deben incrementar su producción científica. Para ello, es necesario que sus integrantes posean recursos cognitivos para efectuar la tarea de investigar, entre los miembros de la universidad, sus estudiantes requieren competencias que les permitan comprender lo leído, sobre todo textos científicos; sin embargo, en las instituciones universitarias se evidencia la existencia de un alto porcentaje de estudiantes carentes de destrezas para la lectura y comprensión de textos (Carlino, 2005).

Diferentes estudios señalan que las competencias lectoras son herramientas indispensables para el aprendizaje, las cuales deben desarrollarse y consolidarse en la etapa pre-escolar y escolar (González, López, Villar y Rodríguez, 2013; Muñoz, Muñoz, García y Granado, 2013; Flórez-Romero y Arias-Velandia, 2010; Muñoz, 2002). A pesar de ello, sistemas educativos como el de Perú no logran desarrollar destrezas lectoras básicas (Ministerio de Educación, Cultura y Deporte [MECD], 2012), de tal forma, los estudiantes que acceden a la educación superior muestran limitaciones, tanto en la velocidad de lectura como en comprensión de textos, lo cual también repercute en su rendimiento académico (Riffo y Contreras, 2012).

Lo mencionado no es una realidad exclusiva del escenario peruano, pues, en otros países latinoamericanos como Chile, Velásquez, Cornejo y Roco (2008) reportan que los estudiantes universitarios muestran un bajo nivel de comprensión lectora. Problemática similar se vive en Colombia según Calderón-Ibáñez y Quijano-Peñuela (2010), quienes proponen como solución realizar intervenciones a nivel de la formación escolar. Sin embargo, ambos estudios no plantean qué hacer con los estudiantes universitarios que ya experimentan las dificultades cognitivas expuestas. Por su parte, Guevara, Guerra, Delgado y Flores (2014) refieren que los estudiantes universitarios mexicanos presentan deficiencias en la destreza lectora, sugiriendo que las instituciones de educación superior adopten medidas para hacer frente a la problemática. El problema señalado muestra un incremento en los últimos años, puesto que el estudiante universitario hace uso de recursos tecnológicos que obstaculizan el adecuado desarrollo de competencias lectoras como lo manifiesta Trillos-Pachecho (2013), quien en base a resultados de una investigación experimental desarrollada con estudiantes colombianos, manifiesta que las nuevas tecnologías originan una lectura hipermedial que guarda relación directa con deficiencias en las destrezas para la comprensión de textos.

Frente a la situación expuesta, surge la necesidad de reflexionar en los contextos universitarios sobre la urgencia de potenciar habilidades lectoras para el aprendizaje (Fernández-Lozano, González-Ballesteros, De-Juanas y Martín, 2011), lo cual ha motivado el desarrollo de algunas investigaciones, a partir de cuyos resultados se han propuesto acciones. Tal es el caso de Martínez-Díaz, Díaz y Rodríguez (2011) quienes

ISL, vol. 8, 2017, págs. 49-59 ISSN: $2340-8685$
Barboza-Palomino, M. y Ventura-León, J.L. (2017): Aplicación de un programa para mejorar la velocidad y comprensión de lectura en estudiantes universitarios, Investigaciones Sobre Lectura, 8, 4959. 
resaltan que los docentes universitarios pueden desempeñar un papel importante como mediadores en el desarrollo de la comprensión lectora, sobre todo de textos científicos.

En relación a los mecanismos que subyacen a la lectura, algunas investigaciones la definen como un proceso activo y complejo de construcción de significados, mediado por un sistema de procesamiento de información que está compuesto por subsistemas relativamente independientes que cumplen una función específica (Cuetos, 1996; LopezHiges, Rubio, Villoria y Mayoral, 2001). De acuerdo con Escurra (2003), la comprensión lectora reside tanto en el lector como en el texto. Por tanto, influyen las claves proporcionadas en el texto, así como el conocimiento previo del lector. Asimismo, LopezHiges, Rubio, Villoria y Mayoral (2001) asumen la comprensión como un proceso constructivo, donde el lector integra representaciones que elabora a partir del texto, las cuales infiere en base a su conocimiento previo.

Así, el proceso de comprensión de lectura está marcado por momentos de comprobación en la que el lector contrasta su modelo mental creado con lo que plantea el texto, de modo que, cuando el modelo no se corresponde con el texto, pone en marcha estrategias de metacomprensión. Por el contrario, cuando no posee un esquema apropiado para comprender conceptos, fracasa (Escurra, 2003). Sí el lector ha adquirido la habilidad, estos procesos se realizan de forma automatizada, desarrollando una lectura fluida; tal es así, que Nutall (1996) sugiere que los estudiantes con estrategias para la comprensión lectora suelen exhibir mayores niveles de velocidad de lectura.

La rapidez de lectura es definida por Aguilar et al. (2010) como la velocidad que presentan las personas para nombrar o reconocer palabras durante un determinado periodo de tiempo. Al respecto, Arias y Justo (2012) añaden que la velocidad de lectura está relacionada con la inteligencia y que a la vez correlaciona positivamente con la comprensión lectora. De otro lado, Silva (2014) menciona que la comprensión lectora no es una destreza que se desarrolle automáticamente con el proceso de decodificación, ya que la persona requiere aprender estrategias que le permita comprender, lo cual irá a la par de la velocidad que tenga al momento de decodificar textos.

Han surgido diversos programas proponiendo actividades para mejorar la velocidad y comprensión de lectura. En el presente estudio se ha utilizado un método clásico de lectura veloz que plantea un conjunto de técnicas que en teoría contribuyen a reducir vicios de lectura, particularmente en los procesos de percepción y concentración, trabajando la visualización global e integración de palabras en frases. La metodología es utilizada por la UPN para incrementar el potencial de velocidad y comprensión de lectura en estudiantes que inician su formación universitaria, asumiendo el supuesto de automatización que plantea el programa, que conllevaría a mejorar los niveles de comprensión. En esa línea, Fajardo, Hernández y González (2012) afirman que la 
automatización del proceso de decodificación garantiza altos niveles de comprensión lectora.

En relación a la efectividad de la metodología de lectura veloz, estudio y memoria utilizada por la UPN no se encuentran estudios que afirmen su utilidad. En términos generales, los programas orientados a desarrollar la comprensión lectora resultan escasos a nivel de la literatura científica. Sin embargo, existen un conjunto de trabajos de tesis, sobre todo en el contexto escolar, que aportan información sobre la ejecución de diferentes propuestas. De otro lado, la literatura científica permite rastrear que se ha incluido la variable comprensión lectora dentro del estudio del desarrollo cognitivo. Es así que, por ejemplo, Merino, Mathiesen, Mora, Castro y Navarro (2014) ejecutaron una investigación para conocer el efecto del Programa Talentos en el desarrollo cognitivo y emocional de escolares chilenos talentosos, encontrando que la primera dimensión (entre ellas la comprensión lectora) se ve afectada positivamente por las actividades desplegadas. Asimismo, estudios efectuados en contexto escolar demostraron cómo la tutoría entre iguales tiene repercusiones en la fluidez y comprensión lectora (Moliner, Flores y Duran, 2011; Valdebenito y Duran, 2013; Duran y Valdebenito, 2014; Valdebenito y Duran, 2015). Por su parte, se visualizan propuestas que se han centrado en la comprensión de textos como medio para desarrollar otros procesos; tal es el caso, que Tolosa y Zerpa (2009) constatan el efecto positivo de una propuesta de comprensión lectora en el razonamiento moral de escolares venezolanos.

Considerando el contexto mencionado y los antecedentes expuestos, el estudio planteó como objetivo: evaluar el efecto de la aplicación del programa de lectura veloz, estudio y memoria en una muestra de estudiantes de la Universidad Privada del Norte (UPN), tras haber aplicado las actividades por un periodo de 16 semanas.

\section{Método}

Se desarrolló una investigación empírica que adoptó la estrategia manipulativa de tipo cuasi experimental longitudinal pretest-postest (Ato, López y Benavente, 2013), ya que en un único grupo se aplicó un material de evaluación para obtener una medida inicial y final en relación a la velocidad y comprensión de lectura, lo cual permitió conocer el efecto del programa aplicado.

\subsection{Participantes}

La muestra del estudio estuvo constituida por 1181 estudiantes distribuidos en 29 grupos, los cuales cursaron estudios en el primer ciclo del semestre académico 2014-II en la UPN. El muestreo fue no probabilístico de tipo intencional, considerando como criterios de inclusión que los estudiantes seleccionados estén matriculados en el semestre académico correspondiente en cualquiera de las asignaturas. Asimismo, se trabajó con

ISL, vol. 8, 2017, págs. 49-59 ISSN: $2340-8685$
Barboza-Palomino, M. y Ventura-León, J.L. (2017): Aplicación de un programa para mejorar la velocidad y comprensión de lectura en estudiantes universitarios, Investigaciones Sobre Lectura, 8, 4959. 
estudiantes de las 4 sedes de la universidad: Lima Centro, Lima Norte, Cajamarca y Trujillo.

\begin{tabular}{|cccc}
\hline Sede & Grupos & Cantidad & \% Total \\
\hline Lima Norte & 10 & 402 & 34 \\
\hline Lima Centro & 5 & 209 & 18 \\
\hline Trujillo & 10 & 405 & 34 \\
\hline Cajamarca & 4 & 165 & 14 \\
\hline Total general & 29 & 1181 & 100.00 \\
\hline
\end{tabular}

Tabla 1. Composición de la muestra de estudiantes del segundo semestre (2014-II) de la UPN

\subsection{Materiales}

Se registró la velocidad y comprensión de lectura utilizando un material de evaluación que consistía en un texto con una determinada cantidad de palabras y su correspondiente cuestionario de preguntas para evaluar la comprensión lectora. Asimismo, se utilizó un cronómetro y ficha de registro de datos por grupo.

\subsection{Procedimiento}

Se determinaron las variables de estudio, fijándose como variable independiente el programa de lectura y como variable dependiente el nivel de velocidad y comprensión de lectura. Posteriormente, se planteó como hipótesis que el programa mejora la velocidad y comprensión lectora; por tanto, para su comprobación se utilizó como prueba de contraste la $t$ de Student, donde la hipótesis nula asume que no existen diferencias estadísticas entre el antes y después de la aplicación del programa.

Después, de forma no probabilística e intencional, se seleccionaron 29 grupos de estudiantes matriculados en el primer ciclo del semestre académico 2014-II de las 4 sedes de la UPN, a quienes se les aplicó el material de evaluación obteniendo un rango inicial de velocidad y comprensión de lectura. El nivel de velocidad de lectura implicó determinar la cantidad de Palabras Leídas por Minuto (PLM), que se obtuvo dividiendo el número de palabras leídas del texto expuesto en el material de evaluación y el tiempo empleado. Por su parte, la comprensión lectora se determinó valorando las Palabras Comprendidas por Minuto (PCM), para lo que se realizó la operación de multiplicación de las PLM con la tasa de comprensión (TC) dividido entre 100.

Las actividades del programa se aplicaron durante 16 semanas. Al término del desarrollo de la propuesta se obtuvo un rango final de velocidad y comprensión de lectura aplicando las fórmulas señaladas anteriormente. Finalmente se calculó el tamaño del efecto con el objetivo de constatar la magnitud práctica de la diferencia estadística en relación a la velocidad y comprensión de lectura a partir de la aplicación del programa, obteniéndose la d de Cohen (Ledesma, Macbeth y Cortada de Kohan, 2008), que implicó 
estimar la diferencia de medias de los promedios de calificaciones de ambos momentos (rango inicial y final) dividido por la desviación estándar.

\section{Resultados}

\subsection{Análisis descriptivo}

Los resultados del análisis de la bondad de ajuste a la curva normal realizado a través de la prueba de Kolmogorov-Smirnov indican que la velocidad de lectura y comprensión lectora son estadísticamente significativas, rechazando de esta forma la hipótesis nula que asume que los datos no difieren de una distribución normal. En función de estos resultados, se decidió utilizar estadísticos paramétricos. Aquello indica que los datos cumplieron con la condición de aproximarse a una distribución normal (Altman, 1991).

\begin{tabular}{cccc}
\hline Variables & Media & D.E. & K-S Z \\
\hline Velocidad de lectura & 147.62 & 35.696 & $.200^{*}$ \\
\hline Comprensión lectora & 177.31 & 37.070 & $.200^{*}$ \\
\hline $\mathbf{N}=\mathbf{1 1 8 1} ; * \mathbf{P}<\mathbf{. 0 0 5}$ & & & \\
\hline
\end{tabular}

Tabla 2. Test de bondad de ajuste a la curva normal de Kolmogorov-Smirnov

\subsection{Análisis comparativo}

El contraste en relación a los niveles de velocidad de lectura y la comprensión lectora producto de la aplicación del programa fue realizado empleando la prueba $t$ de Student de diferencias de medias para grupos emparejados, observándose en la tabla 3 la existencia de diferencias estadísticamente significativas en relación a la velocidad de lectura $(\mathrm{t}=3.242 ; \mathrm{p}<.005)$. Además, se constata una diferencia práctica moderada como lo indica la medida de tamaño del efecto $(\mathrm{d}=.79)$, afirmándose que el programa aplicado generó un efecto moderado en la mejora de la velocidad de lectura de los participantes.

Por otra parte, en la tabla 3 también se aprecia que no existen diferencias estadísticamente significativas en relación a la comprensión de lectura $(\mathrm{t}=-1.077 ; \mathrm{p}<$ .005). Asimismo, se verifica que el programa aplicado no tiene repercusiones prácticas significativas en la mejora de la comprensión lectora de los participantes, como lo señala la medida del tamaño del efecto $(d=.28)$, que resulta pequeña.

\begin{tabular}{|c|c|c|c|c|c|c|c|}
\hline \multirow[t]{2}{*}{ Variables } & \multicolumn{2}{|c|}{ Pre test } & \multicolumn{2}{|c|}{ Post test } & \multirow[t]{2}{*}{$\mathbf{t}$} & \multirow{2}{*}{$\begin{array}{l}\text { Sig. } \\
(\mathrm{z})\end{array}$} & \multirow[t]{2}{*}{$d$} \\
\hline & Media & D.E. & Media & D.E. & & & \\
\hline Velocidad de lectura & 147.62 & 35.696 & 209.97 & 104.559 & 3.242 & $.003 *$ & .79 \\
\hline Comprensión lectora & 177.31 & 37.070 & 160.03 & 78.293 & -1.077 & .290 & .28 \\
\hline $\begin{array}{l}\mathrm{N}=1181 ; * \mathrm{p}<.05 \\
d \text { de Cohen (tamaño d }\end{array}$ & ecto) & & & & & & \\
\hline
\end{tabular}

Tabla 3. Prueba t de Student y tamaño del efecto

ISL, vol. 8, 2017, págs. 49-59 ISSN: $2340-8685$
Barboza-Palomino, M. y Ventura-León, J.L. (2017): Aplicación de un programa para mejorar la velocidad y comprensión de lectura en estudiantes universitarios, Investigaciones Sobre Lectura, 8, 4959. 


\section{Discusión y conclusiones}

La carencia de competencias para la comprensión lectora es un común denominador en diferentes escenarios latinoamericanos de educación superior (Carlino, 2005; Velásquez et al., 2008; Calderón-Ibánez y Quijano-Peñuela, 2010; Guevara, 2014). El Perú no es ajeno a ello, como lo afirma el MECD (2012): los estudiantes que acceden a la universidad muestran pobres destrezas lectoras. En dicho contexto, la UPN como parte de sus políticas educativas desarrolla intervenciones dirigidas a la mejora de la velocidad y comprensión de textos, sustentados en que la automatización del proceso de decodificación (velocidad de lectura) permitirá lograr altos niveles de comprensión lectora, tal como lo señalan Fajardo et al. (2012).

En función a lo señalado, la presente investigación tuvo por objetivo evaluar el efecto de la aplicación de un programa basado en la mejora de la velocidad y comprensión de lectura de estudiantes del primer ciclo de la UPN que cursaron estudios en el semestre académico 2014-II. Los resultados de la diferencia estadística y magnitud del efecto evidencian que la propuesta tuvo un efecto positivo moderado real en el incremento de la velocidad de lectura de los participantes. Por su parte, en relación a la comprensión de lectura, la diferencia estadística y medida del tamaño del efecto indican que el programa no resultó efectivo.

Partiendo de lo expuesto en el párrafo anterior, los resultados refutan lo mencionado por Fajardo et al. (2012) y Arias y Justo (2012) en relación a la determinación de mejora del nivel de comprensión a partir del incremento de la velocidad de lectura, ya que el aumento de la velocidad no necesariamente implica un mayor nivel de comprensión de lo leído. Por ello, los hallazgos del estudio se alinean a lo propuesto por Silva (2014), en el hecho de catalogar a la comprensión lectora como una destreza que no se desarrolla automáticamente con la decodificación, y que obedece al dominio de estrategias de comprensión. Asimismo, se desprende como tarea pendiente para futuras investigaciones probar el supuesto teórico de Nutall (1996) que refiere una relación de determinación inversa entre velocidad y comprensión de lectura.

De igual forma, lo encontrado resalta el carácter de proceso complejo y constructivo que se atribuye a la comprensión lectora (Cueto, 1996; Lopez-Higes et al., 2001; Escurra, 2003), presentándose como una necesidad romper las fronteras del escenario de reflexión propuesto por Fernández-Lozano et al. (2011), concibiendo a las destrezas lectoras como herramientas cognitivas que se tienen que desarrollar, consolidar y ajustar en todo el proceso educativo. Si bien es cierto que se afirma que el desarrollo y consolidación de las competencias lectoras corresponde a la etapa pre-escolar y escolar (González et al., 2013; Flores-Romero y Arias-Velandia, 2010; Muñoz, 2002), cabe resaltar que un estudiante universitario se enfrenta a textos de mayor complejidad, sobre todo científicos, frente a los cuales requiere ajustar sus estrategias de comprensión lectora. 
La investigación no contempló variables como el rendimiento académico, por lo cual no se puede describir a partir de los resultados la relación existente entre velocidad y comprensión de lectura con el desempeño académico, quedando pendiente corroborar lo señalado por Riffo y Contreras (2012). Asimismo, no se consideró la variable uso de nuevas tecnologías y lectura hipermedial como causantes de deficientes destrezas lectoras tal como lo demostró Trillos-Pacheco (2013). Finalmente, tampoco se trabajó el supuesto de Martínez-Díaz et al. (2011) en relación a la influencia de la labor docente en el desarrollo de competencias lectoras.

El contraste de los hallazgos con resultados de otros estudios resulta complicado por la ausencia de literatura científica que reporte investigaciones de características similares. Sin embargo, existen algunos informes científicos en el contexto escolar que ayudan a entender la importancia de la comprensión lectora y definir nuevas rutas de investigación. Tal es el caso de Merino et al. (2014) que afirman que actividades donde se fomenta la comprensión lectora afectan positivamente al desarrollo cognitivo, afirmación que no se sometió a comprobación en el presente estudio. Por su parte, se informa de que la tutoría entre iguales repercute en la velocidad y comprensión de textos (Moliner et al., 2011; Valdebenito \& Duran, 2013; Duran y Valdebenito, 2014; Valdebenito y Duran, 2015), situándose la metodología empleada como alternativa para elaborar y someter a prueba la efectividad de nuevos programas de lectura. Asimismo cabe considerar que Tolosa y Zerpa (2009) mencionan que la comprensión lectora influye en el razonamiento moral, tema que puede ser abordado en futuras investigaciones.

El programa elaborado en base a una metodología de lectura veloz, estudio y memoria en la práctica no logró el objetivo de incrementar de forma significativa la velocidad y comprensión de lectura de los participantes. Por ende, a partir de los resultados del estudio, se sugiere replantear las decisiones institucionales en materia de intervención educativa en la UPN, en particular, la metodología usada en la elaboración de programas de lectura. Asimismo, permite orientar futuras prácticas y metodologías a seguir por instituciones universitarias e investigadores que apunten a desarrollar destrezas lectoras en estudiantes. Finalmente, los hallazgos facilitan la reflexión sobre la importancia de la toma de decisiones en base a la evidencia científica.

\section{Referencias}

Aguilar, M., Navarro, J. I., Menacho, I., Alcale, C., Marchena, E. \& Olivier, P. R. (2010). Velocidad de nombrar y conciencia fonológica en el aprendizaje inicial de la lectura. Psicothema, 22(3), 436-442.

Altman, D. G. (1991). Estadística práctica para la investigación médica. Londres: Chapman y Hall. programa para mejorar la velocidad y comprensión de lectura en estudiantes universitarios, Investigaciones Sobre Lectura, 8, 4959. 
Arias, W. L. \& Justo, O. (2012). Velocidad de lectura e inteligencia en estudiantes de ingeniería. Revista de Psicología, 2(1), 43-55.

Ato, M., López, J. \& Benavente, A. (2013). Un sistema de clasificación de los diseños de investigación en psicología. Anales de Psicología, 29(3), 1038-1059.

Calderón-Ibáñez, A. \& Quijano-Peñuela, J. (2010). Características de comprensión lectora en estudiantes universitarios. Estudios Socio-Jurídicos, 12(1), 337-364.

Carlino, P. (2005). Escribir, leer y aprender la universidad: una introducción a la alfabetización académica. Buenos Aires: Fondo de Cultura Académica de Argentina.

Cuetos, F. (1996). Psicología de la lectura. Diagnóstico y tratamiento de los trastornos de lectura. Getafe: Editorial Escuela.

Duran, D. \& Valdebenito, V. (2014). Desarrollo de la competencia lectora a través de la tutoría entre iguales como respuesta a la diversidad del alumnado. Revista Latinoamericana de Educación Inclusiva, 8(2), 141-160.

Escurra, M. (2003). Comprensión de lectura y velocidad lectora en alumnos de sexto grado de primaria de centros educativos estatales y no estatales de Lima. Persona: Revista de la Facultad de Psicología, (6), 99-134.

Fajardo, A., Hernández, J. \& González, A. (2012). Acceso léxico y comprensión lectora: un estudio con jóvenes universitarios. Revista Electrónica de Investigación Educativa, 14(2), 25-33 Recuperado el 4 de agosto, 2016 de: http://redie.uabc.mx/redie/article/viewFile/305/468

Fernández-Lozano, M. P., González-Ballesteros, M., De-Juanas, A. \& Martín, R. (2011). Reflexión sobre la potencialidad para el aprendizaje de las tareas de lecto-escritura requeridas a estudiantes universitarios. Revista de Psicología y Educación, (6), 111-120.

Flórez-Romero, R. \& Arias-Velandia, N. (2010). Evaluación de conocimientos previos del aprendizaje inicial de lectura. Magis, Revista Internacional de Investigación en Educación, 2(4), 329-344.

González, R. M., López, S., Villar, J. \& Rodríguez, A. (2013). Estudio de los predictores de la lectura. Revista de Investigación en Educación, 11(2), 98-110.

Guevara, Y., Guerra, J., Delgado, U. \& Flores, C. (2014). Evaluación de distintos niveles de comprensión lectora en estudiantes mexicanos de psicología. Acta Colombiana de Psicología, 17(2), 113-121. 
Ledesma, R., Macbeth, G. \& Cortada de Kohan, N. (2008). Tamaño del efecto: revisión teórica y aplicaciones con el sistema estadístico ViSta. Revista Latinoamericana de Psicología, 40(3), 425-439.

Lopez-Higes, R., Rubio, S., Villoria, C. \& Mayoral, J. A. (2001). Exploración cognitiva de la lectura I: presentación de un nuevo instrumento. Revista de Psicología General y Aplicada, 54(3), 467-496.

Martínez-Díaz, E. S., Díaz, N. \& Rodríguez, D. E. (2011). El andamiaje asistido en procesos de comprensión lectora en universitarios. Educación y Educadores: 14(3), 531-556.

Merino, J. M., Mathiesen, M. E., Mora, O., Castro, G. \& Navarro, G. (2014). Efectos del Programa Talentos en el desarrollo cognitivo y socioemocional de sus alumnos. Estudios Pedagógicos, 40(1), 197-214.

Ministerio de Educación, Cultura y Deporte (2012). Panorama de la educación. Indicadores de la OCDE. Recuperado el 6 de agosto, 2016 de: http://www.mecd.gob.es/dctm/inee/internacional/panorama2012.pdf?documentI $\underline{\mathrm{d}=0901 \mathrm{e} 72 \mathrm{~b} 81415 \mathrm{~d} 28}$

Moliner, L., Flores, M. \& Duran, D. (2011). Efectos sobre la mejora de las competencias lingüísticas y la autoimagen lectora a través de un programa de tutoría entre iguales. Revista de Investigación en Educación, 9(2), 209-222.

Muñoz, C. (2002). Aprendizaje de la lectoescritura y conciencia fonológica: un enfoque psicolingüístico del proceso de alfabetización inicial. Psykhe, 11(1), 29-42.

Muñoz, E. M., Muñoz, L.M., García, M.C. \& Granado, L. A. (2013). La comprensión lectora de textos científicos en el proceso de enseñanza-aprendizaje. Humanidades Médidas, 13(3), 772-804.

Nuttall, C. (1996). Teaching reading skills in a foreing language. Oxford: Heinemann English Language Teaching.

Riffo, B. \& Contreras, M. (2012). Experiencia académica y comprensión de textos especializados en estudiantes universitarios de Ciencias Políticas. Estudios Pedagógicos, 38(2), 201-219.

Silva, M. (2014). El estudio de la comprensión lectora en Latinoamérica: necesidad de un enfoque en la comprensión. Innovación Educativa, 14(64), 47-55.

Tolosa, A. D. \& Zerpa, C. E. (2009). Efecto de un programa de comprensión de textos de contenido moral en las estructuras de razonamiento moral de estudiantes del primer año del Ciclo Diversificado. Revista de Pedagogía, 30(87), 247-280. programa para mejorar la velocidad y comprensión de lectura en estudiantes universitarios, Investigaciones Sobre Lectura, 8, 4959. 
Trillos-Pacheco, J. J. (2013). La lectura hipermedial y su incidencia en la comprensión lectora en estudiantes universitarios. Palabra Clave, 16(3), 944-992.

Valdebenito, V. \& Duran, D. (2013). La tutoría entre iguales como un potente recurso de aprendizaje entre alumnos: efectos, fluidez y comprensión lectora. Perspectiva Educacional, 52(2), 154-176.

Valdebenito, V. \& Duran, D. (2015). Formas de interacción implicadas en la promoción de estrategias de comprensión lectora a través de un programa de tutoría entre iguales. Revista Latinoamericana de Psicología, 47(2), 75-85.

Velásquez, M., Cornejo, C. \& Roco, A. (2008). Evaluación de la competencia lectora en estudiantes de primer año de carreras del Área Humanista y carreras de la salud en tres universidades del Consejo de Rectores. Estudios Pedagógicos: 34(1), 123138. 